\title{
PROPOSTA DE PASSOS PARA A SEGURANÇA DO PACIENTE NO ATENDIMENTO PRÉ-HOSPITALAR MÓVEL ${ }^{1}$
}

\author{
Grayce Louyse Tinôco de Castro², Francis Solange Vieira Tourinho ${ }^{3}$, Maria de Fátima da Silva Vieira Martins ${ }^{4}$, \\ Kleyton Santos de Medeiros ${ }^{5}$ Patricia Ilha ${ }^{6}$, Viviane Euzébia Pereira Santos ${ }^{7}$
}

${ }^{1}$ Artigo extraído dissertação - Atendimento pré-hospitalar móvel: mapeando riscos e prevenindo erros, apresentada ao Programa de Pós-Graduação em Enfermagem da Universidade Federal do Rio Grande do Norte (UFRN), em 2013.

${ }^{2}$ Mestre em Enfermagem. Programa de Pós-Graduação em Enfermagem da UFRN. Enfermeira do Samu Natal. Natal, Rio Grande do Norte, Brasil. E-mail: graycelouyse@yahoo.com.br

${ }^{3}$ Doutora em Saúde da Criança e do Adolescente. Professora do Departamento de Enfermagem e do Programa de Pós-Graduação em Enfermagem da Universidade Federal de Santa Catarina (UFSC). Florianópolis, Santa Catarina, Brasil. E-mail: francis.tourinho@ufsc.br

${ }^{4}$ Doutora em Sociologia. Professora da Escola Superior de Enfermagem da Universidade do Minho. Portugal. E-mail: fmartins@ ese.uminho.pt

${ }^{5}$ Enfermeiro da Liga Contra o Câncer. Natal, Rio Grande do Norte, Brasil. E-mail: kleyton_medeiros@hotmail.com

${ }^{6}$ Doutoranda em Enfermagem. Programa de Pós-Graduação em Enfermagem da UFSC. Florianópolis, Santa Catarina, Brasil. E-mail: ilha.patricia@gmail.com

${ }^{7}$ Doutora em Enfermagem. Professora do Departamento de Enfermagem e do Programa de Pós-Graduação em Enfermagem da UFRN. Natal, Rio Grande do Norte, Brasil. E-mail: vivianeepsantos@gmail.com

\section{RESUMO}

Objetivo: propor passos para a segurança do paciente a partir da análise dos riscos no atendimento pré-hospitalar móvel sob a ótica dos enfermeiros.

Método: estudo quantitativo, e descritivo. Amostra intencional, composta por 23 enfermeiros. Utilizou-se o método de pesquisa fotográfica para identificar os riscos à segurança do paciente em um Serviço de Atendimento Móvel de Urgência de uma cidade do Rio Grande do Norte. A coleta foi realizada de março a junho de 2012 e organizada em cinco etapas: obtenção das fotos de situações de segurança e não segurança; seleção de fotos pela qualidade de imagem; seleção de fotos pelos experts da temática; seleção de dez imagens de modo aleatório; e aplicação do instrumento de análise das fotos pelos profissionais. Foi utilizada a análise de conteúdo e análise descritiva.

Resultados: os riscos apontados no estudo foram: dificuldades no acondicionamento de equipamentos e materiais; especificidades do trabalho no atendimento pré-hospitalar móvel; risco de infecção; risco de traumas; e dificuldades na administração de medicamentos. A partir dessas informações e confrontos com a literatura, foram sugeridos dez passos para a segurança do paciente no atendimento préhospitalar, voltados às necessidades de redução dos riscos apresentados.

Conclusão: a análise de riscos e proposta de intervenções para a segurança do paciente favorecem a qualidade do atendimento em saúde, com benefícios na esfera: paciente, equipe, profissional e ambiente. Sugere-se que sejam desenvolvidas de acordo com as necessidades de cada serviço.

DESCRITORES: Segurança do paciente. Qualidade da assistência à saúde. Gestão de riscos. Assistência pré-hospitalar. Serviços médicos de urgência. Fotografia.

\section{PROPOSAL FOR STEPS TOWARDS PATIENT SAFETY IN MOBILE EMERGENCY CARE}

\begin{abstract}
Objective: propose steps for patient safety based on the analysis of risks in mobile emergency care from nurses' perspective.

Method: quantitative and descriptive study. Intentional sample consisting of 23 nurses. The photographic research method was used to identify the risks for patient safety in a Mobile Emergency Care Service in a city in Rio Grande do Norte (Brazil). The data were collected between March and June 2012 and organized in five phases: collection of pictures from safe and non-safe situations; selection of pictures based on the image quality; selection of pictures by experts on the theme; random selection of ten images; and application of picture analysis tool by the professionals. Content analysis and descriptive analysis were used.

Results: the risks appointed in the study were: difficulties to store equipment and material; particularities of work in mobile emergency care; risk of infection; risk of traumas; and medication administration difficulties. Based on this information and confrontations with the literature, ten steps were suggested towards patient safety in emergency care, focused on the needs to reduce the risks presented.

Conclusion: the risk analysis and the intervention proposal towards patient safety favor the quality of health care, with benefits in the following spheres: patient, team, equipment, professional and environment. These should be developed in accordance with the needs of each service.
\end{abstract}

DESCRIPTORS: Patient safety. Quality of health care. Risk management. Prehospital care. Emergency medical services. Photography. 


\title{
PROPUESTA DE PASOS A SEGUIR PARA LA SEGURIDAD DEL PACIENTE EN LA ATENCIÓN PRE-HOSPITALARIA MÓVIL
}

\begin{abstract}
RESUMEN
Objetivo: proponer los pasos a seguir para la seguridad del paciente a partir del análisis de los riesgos en la atención pre-hospitalaria móvil y bajo la óptica de los enfermeros.

Método: estudio cuantitativo, y descriptivo. Muestra intencional compuesta por 23 enfermeros. Se utilizó el método de investigación fotográfica para identificar los riesgos para la seguridad del paciente en un Servicio de Atención Móvil de Urgencia de una ciudad del estado de Rio Grande do Norte. La recolección se realizó de Marzo a Junio del 2012 y se organizó en cinco etapas: obtención de las fotos de situaciones de seguridad y sin seguridad, selección de fotos por la cualidad de imagen, selección de fotos por los peritos en la temática, selección de diez imágenes de forma aleatoria y aplicación del instrumento de análisis de las fotos por los profesionales. Se usó el análisis del contenido y el análisis descriptivo.
\end{abstract}

Resultados: los riesgos mencionados en el estudio fueron las dificultades en el acondicionamiento de equipamientos y materiales, especificidades del trabajo en la atención pre-hospitalaria móvil, riesgo de infección, riesgo de traumas y dificultades en la administración de medicamentos. A partir de estas informaciones y cotejos con la literatura fueron sugeridos diez pasos a seguir para la seguridad del paciente en la atención pre-hospitalaria relacionados con las necesidades de reducción de los riesgos presentados.

Conclusión: el análisis de riesgos y propuesta de intervenciones para la seguridad del paciente favorece la cualidad de la atención en la salud y con beneficios en las áreas paciente, equipo, profesional y ambiente. Se sugiere que sean desarrolladas de acuerdo con las necesidades de cada servicio.

DESCRIPTORES: Seguridad del paciente. Cualidad de la asistencia para la salud. Gestión de riesgos. Asistencia pre-hospitalaria. Servicios médicos de urgência. Fotografía.

\section{INTRODUÇÃO}

O conceito de segurança do paciente refere-se à prevenção de possíveis danos causados durante a realização de cuidados à saúde dos pacientes e demais sujeitos envolvidos nesse processo, que estão sempre susceptíveis a uma cadeia de erros, devido à complexidade dos fatores que envolvem o cuidado. Para que essa prevenção seja alcançada de forma efetiva, algumas estratégias básicas da segurança do paciente precisam ser implementadas, como a formação profissional para segurança, a conscientização organizacional e institucional, a distribuição de recursos e a atualização constante dos processos, com uma avaliação crítica, para construção de um sistema propício para o desenvolvimento de uma cultura de segurança positiva e estruturada para a diminuição de riscos e prevenção dos erros. ${ }^{1}$

De acordo com a especificidade de cada setor e das necessidades dos profissionais que trabalham no serviço, assim como as diferentes ações que são executadas, cada unidade deve elaborar um plano ou diretriz que se adeque a essas necessidades e ao perfil de seus profissionais, com a participação e construção conjunta, e articulada da tríade, governo, serviço e ensino. ${ }^{2}$

$\mathrm{O}$ atendimento pré-hospitalar (APH), por ser uma área com amplos conhecimentos e de específica atuação, tem exigido dos profissionais uma formação que seja compatível com a realidade dos atendimentos solicitados pela população, eesse atendimento engloba várias classes profissionais com práticas e saberes específicos, formando equipes inter-relacionadas de forma coletiva, resultando em atos desenvolvidos por cada um deles e por todos, devendo estar preparadas para assistir qualquer tipo de agravo em todas as faixas etárias. Assim, o conhecimento técnico é o dominante de todos e transcorre todas as categorias, considerando-se as especificidades, competências e responsabilidades de cada membro da equipe. ${ }^{3}$

Uma das características dos serviços de APH móveis é que eles realizam o atendimento precoce à vítima, após ter ocorrido um agravo à sua saúde também de natureza clínica, cirúrgica, traumática e psiquiátrica, que possa levar ao sofrimento, sequelas ou mesmo à morte, sendo necessário, portanto, prestar-lhe atendimento e/ ou transporte adequado a um serviço de saúde devidamente hierarquizado, de modo que seja prestado um serviço rápido, de qualidade e segurança à população. ${ }^{4}$

Atingir um índice de qualidade na assistência do atendimento pré-hospitalar consiste em não agravar o estado de saúde do paciente até sua chegada ao ambiente de tratamento definitivo, normalmente, o hospital, minimizando complicações e buscando estabilidade hemodinâmica em menor tempo possível. ${ }^{5}$

Os cuidados de saúde inseguros resultam em expressiva morbimortalidade evitável, gastos adicionais com a manutenção dos sistemas de saúde e representam uma grande preocupação na atualidade. ${ }^{6}$ Por outro lado, cuidados e vigilância de alta qualidade permitem que muitos resultados adversos desapareçam, sendo construídos sob os conhecimentos fornecidos pela qualidade da investigação e a análise dos cuidados produzida anteriormente, gerando, assim, uma cultura de segurança. ${ }^{7}$ 
Considerando que o paciente submetido aos serviços de saúde corre o risco de sofrer danos relacionados à assistência, a Organização Mundial da Saúde criou, em 2004, a Aliança Mundial pela Segurança do Paciente, com a missão de coordenar, disseminar e acelerar melhorias para segurança do paciente em termos mundiais. ${ }^{6-9}$ Embora essas ações sejam diretrizes para qualidade na segurança do paciente, ainda não são definidos por instituições internacionais planos de ações diretamente voltados ao atendimento pré-hospitalar, pois são planos de abrangência mais gerais.

Profissionais do Conselho Regional de Enfermagem de São Paulo (COREN-SP), com experiência acumulada na assistência, no ensino e/ou na pesquisa, e muitos anos de dedicação à área da saúde, elaboraram os dez passos para a segurança do paciente, com base em evidências científicas atualizadas, e procuraram apresentá-los de forma objetiva e prática. São eles: 1) identificação do paciente. 2) cuidado limpo e cuidado seguro - higienização das mãos. 3) cateteres e sondas - conexões corretas. 4) cirurgia segura. 5) sangue e hemocomponentes administração segura. 6) paciente envolvido com sua própria segurança. 7) comunicação efetiva. 8) prevenção de queda. 9) prevenção de úlcera por pressão. 10) segurança na utilização de tecnologia. ${ }^{10}$ Garantir a segurança de todos que entram em contato com serviços de saúde é um dos desafios mais importantes que o cuidado em saúde enfrenta hoje, ${ }^{11}$ fato que a torna uma séria questão de saúde global. A identificação, análise e gerência de riscos relacionados com o paciente e possíveis incidentes permitem realizar um cuidado mais seguro a este e minimizar danos. ${ }^{12}$

Nesse contexto, é possível entender que a atuação dos órgãos e profissionais de saúde para a melhoria da qualidade dos serviços, utilizando estratégias, como a criação de passos para a segurança do paciente, estimula reflexões acerca de práticas seguras e colabora para uma ação eficaz. Contudo, observa-se que, na área pré-hospitalar nacional, são escassas as pesquisas específicas sobre o tema.

Encontra-se em estudos internacionais, que buscam estratégias de inserção de práticas seguras no atendimento pré-hospitalar, que eles utilizam da pesquisa de riscos e práticas inseguras, e como o preconizado pela Organização Mundial da Saúde, partem de intervenções de segurança que sejam voltadas para o contexto e necessidade do ambiente e dos profissionais, e também criticam a necessidade de orientações para segurança do paciente voltadas especificamente para o $\mathrm{APH}$, pois a insalubridade e hostilidade do ambiente onde as ações são realizadas requerem também orientações específicas e divergentes dos outros serviços em saúde. ${ }^{13}$

Outros achados também procuraram desenvolver processos seguros durante o atendimento pré-hospitalar, mas com ações mais direcionadas a uma categoria profissional e não ao trabalho multiprofissional que exige a atividade pré-hospitalar. Eles também deixam a crítica de que por mais que existam protocolos que orientem a prática pré-hospitalar, faltam diretrizes diretamente relacionadas à segurança do paciente. ${ }^{14-15}$

Embora existam literaturas internacionais que fundamentem alguns passos para segurança, as necessidades, recursos e contextos são diferentes dos vivenciados no Brasil, necessitando assim de pesquisas nacionais que analisem e sugiram intervenções para melhoria da segurança do paciente. Desta forma, a pesquisa objetivou propor passos para a segurança do paciente a partir da análise dos riscos no atendimento pré-hospitalar móvel sob a ótica dos enfermeiros.

\section{MÉTODO}

Trata-se de estudo descritivo, de abordagem quantitativa. A amostra da pesquisa deu-se de forma intencional através do convite a 28 enfermeiros que trabalhavam no Serviço de Atendimento Móvel de Urgência (SAMU) em um município do Rio Grande do Norte e atuavam em Unidades de Suporte Avançado de Vida, e cumpriam os critérios de participação, dos quais 23 aceitaram participar da pesquisa, constituindo a amostra final.

Como critério de inclusão, considerou-se o tempo de experiência para especialistas recomendado pela Associação Brasileira de Enfermagem em Terapia Intensiva (ABENTI), com base na resolução COFEN n. 389/2011, ${ }^{16}$ que atualiza os procedimentos para registro de especialidades na enfermagem, e que tenham realizado ao menos um curso de formação específica na área de atendimento.

A coleta de dados ocorreu na sede do SAMU, nos meses de março a junho de 2012, e foi organizada em cinco etapas, utilizando-se do método de análise fotográfica, o qual permite a visualização real dos dados obtidos para realizar uma avaliação válida e confiável dos fatores organizacionais que possam influenciar na segurança do paciente, já que as fotografias digitais foram capturadas no local e ambiente de trabalho dos participantes. ${ }^{17}$

A coleta foi constituída das seguintes etapas: $1^{\text {a) }}$ foram coletadas aleatoriamente várias imagens 
através de fotografias digitais de ambientes e ações, sendo estas seguras e/ou não seguras, juntamente com a colaboração dos participantes do estudo; $2^{\mathrm{a}}$ ) foram eliminadas as fotos tremidas ou sem outras condições de uso; $3^{\mathrm{a}}$ ) foram enviadas as imagens coletadas para experts em segurança do paciente para seleção das imagens a serem utilizadas na pesquisa; sendo escolhido um total de 22 fotografias; $4^{\text {a }}$ ) através da função do Microsolft Excel 2010, foram selecionadas aleatoriamente as dez imagens que seriam utilizadas no trabalho; e $5^{\mathrm{a}}$ ) as imagens foram projetadas aos participantes em um computador e eles preencheram um instrumento para cada uma das dez imagens - o Instrumento de Pontuação de Fotografia Digital, o qual era composto de três questões abertas que avaliavam: condição geral da segurança da área fotografada em relação aos procedimentos invasivos; avaliação geral da segurança da área fotografada em relação à utilização dos equipamentos de proteção individual; e um espaço livre para comentários sobre a fotografia. Utilizou-se também uma questão fechada, a qual demanda uma nota de 1 a 10, de acordo com a representatividade da imagem para segurança do ambiente. ${ }^{17}$

A análise dos dados partiu da técnica de análise do conteúdo ${ }^{18}$ para categorização das respostas abertas e, em seguida, foram realizadas análises descritivas dos conteúdos encontrados.

A pesquisa respeitou os aspectos éticos, de acordo com a Resolução n. 466/12, e foi aprovada no Comitê de Ética em Pesquisa da Universidade Federal do Rio Grande do Norte, com certificado de apresentação para apreciação ética CAAE: 0122.0.051.000-11. ${ }^{19}$

\section{RESULTADOS}

Os resultados revelaram diversos riscos identificados pelos enfermeiros nas fotografias, não havendo uma linearidade nas respostas. Após sucessivas leituras dessas observações, foram realizadas categorizações de temas. ${ }^{18}$ Inicialmente, emergiram 12 categorias. Em seguida, realizou-se uma nova categorização, totalizando cinco categorias. Os símbolos C1, C2, C3, C4 e C5 foram utilizados para sua identificação e estas foram analisadas a luz dos dez passos para a segurança do paciente preconizados pelo COREN/SP,${ }^{10}$ fundamentação teórica utilizada para o desenvolvimento das propostas de passos para a segurança do paciente no APHM.

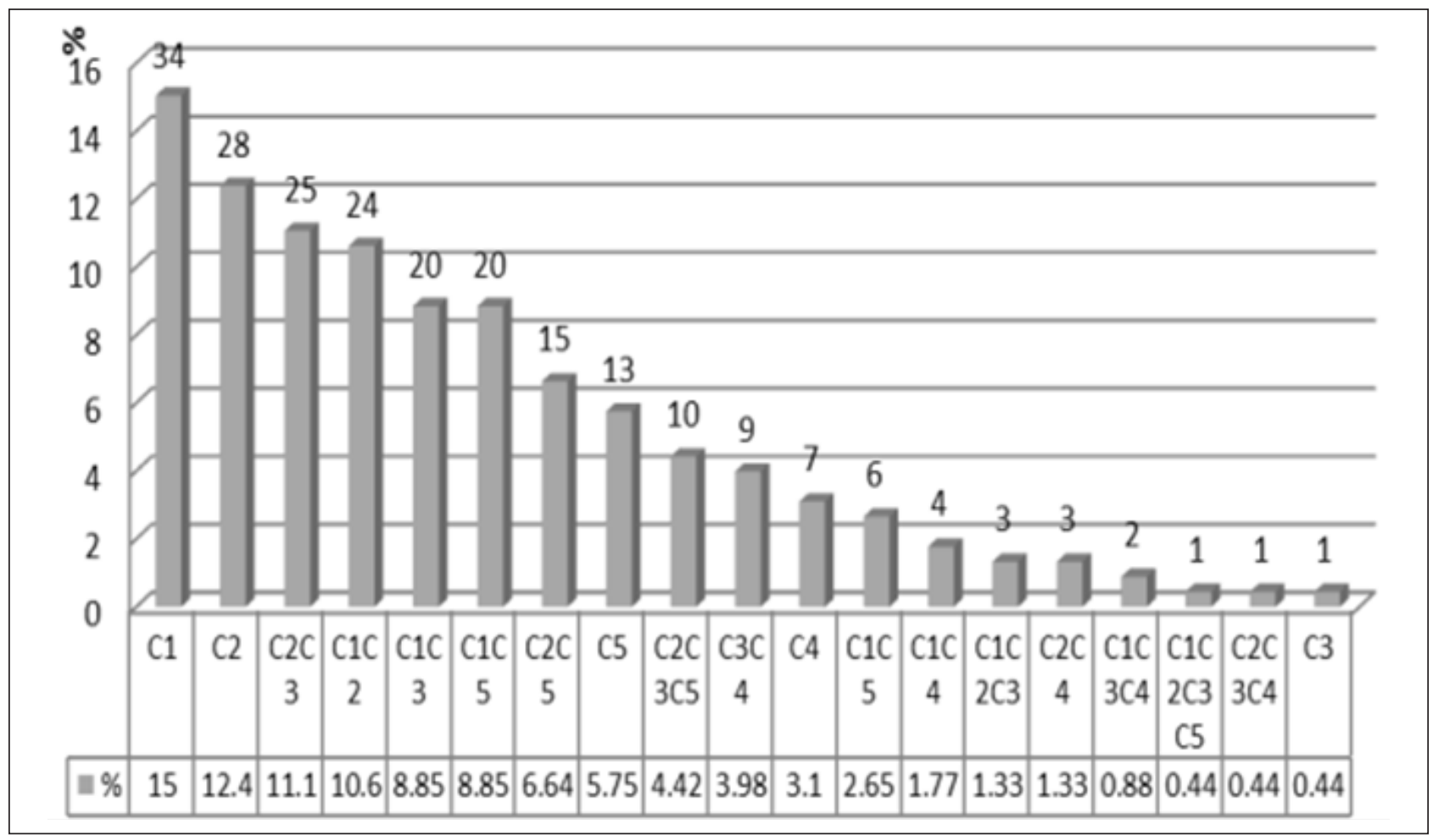

C1 - Dificuldades no acondicionamento de equipamentos e materiais; C2 - Especificidades do trabalho no APHM; C3 - Risco de infecção; C4- Risco de traumas; C5 - Dificuldades na administração de medicamentos.

Figura 1 - Distribuição de categorias de riscos encontradas nas 230 respostas, total de 19 combinações de respostas possíveis. Natal, RN, 2013 
As 230 respostas obtidas nos resultados refletiram diferentes aspectos para os ambientes fotografados e apontam que os temas mais abordados isoladamente por foto, foram: dificuldades no acondicionamento de equipamentos e materiais (C1) com 15\%; especificidades do trabalho no APHM (C2) com 12,4\%; combinações de especificidades do trabalho no APHM e risco de infecção $(\mathrm{C} 2, \mathrm{C} 3) \mathrm{com}$ 11,1\%; e combinações de dificuldades no acondicionamento de equipamentos e materiais e especificidades do trabalho no APHM (C1, C2) 10,6\%, (Figura 1). Ainda na figura 1 , o item dificuldades na administração de medicamentos (C5) aparece isolado com $5,75 \%$, como também combinado a mais cinco possibilidades. Com relação ao risco de traumas e quedas (C4), ele aparece em combinação com risco de infecção (C3) 3,98\%, bem como isoladamente com $3,1 \%$, o que não significa que não apresenta importância, visto que o trabalho no APHM oferece risco de queda pelo atendimento, especialmente, pela trepidação das ambulâncias em movimento.

Baseando-se nestas categorias de riscos evidenciados pelos enfermeiros participantes do estudo e nos dez passos para segurança do paciente, ${ }^{10}$ elaborou-se uma proposta de dez passos para a segurança do paciente no APHM, visando o direcionamento dos profissionais de área tão específica e a melhoria contínua da segurança através do conhecimento e prevenção dos riscos. O quadro 1 apresenta relação de semelhança dos dez passos para a segurança do paciente ${ }^{10} \mathrm{com}$ as categorias de riscos observadas no estudo.

Quadro 1 - Relação de semelhança entre os dez passos para segurança do paciente e as categorias de riscos observadas no estudo. Natal, RN, 2013

\begin{tabular}{|l|l|}
\hline Dez passos para a segurança do paciente & Categorias de riscos observadas no estudo \\
\hline $\begin{array}{l}\text { Passo 2: cuidado limpo é cuidado seguro - } \\
\text { higienização das mãos. }\end{array}$ & $\begin{array}{l}\text { (C3) utilização de Equipamentos de Proteção } \\
\text { Individual (EPIS's); higienização das mãos; risco de } \\
\text { contaminação/infecção }\end{array}$ \\
\hline Passo 3: cateteres e sonda - conexões corretas. & $\begin{array}{l}\text { (C5) dificuldades relacionadas à administração de } \\
\text { medicamentos }\end{array}$ \\
\hline Passo 7: comunicação efetiva. & (C2) especificidades do trabalho no APHM \\
\hline Passo 8: prevenção de queda. & $\begin{array}{l}\text { (C4) quedas e imobilização; risco de traumas e } \\
\text { quedas }\end{array}$ \\
\hline
\end{tabular}

Verifica-se também, na figura 2, que não emergiram categorias de riscos relacionadas diretamente a alguns passos do COREN-SP. ${ }^{10}$ Isto se deve ao fato de os entrevistados não terem feito referência direta a riscos que equivalessem a um dos passos para a segurança do paciente. Entretanto, evidenciaramse riscos específicos ao APHM, como dificuldades no acondicionamento de equipamentos e materiais (C1), além de outras especificidades do trabalho no APHM (C2). Relacionando-se as semelhanças entre os passos e os riscos evidenciados e analisando os passos não avaliados, porém relevantes, sugere-se, no quadro 2 a seguir, as propostas de passos para a segurança no atendimento pré-hospitalar móvel.

\section{Quadro 2 - Dez passos para a segurança do paciente no APHM. Natal, RN, 2013}

Propostas de passos para a segurança do paciente no APHM

Passo 1: identificar o paciente por meio de pulseira, com cores que sinalizem sua gravidade, principalmente nos casos de múltiplas vítimas.

Passo 2: segurança relacionada à higienização das mãos.

Passo 3: segurança na administração de medicamentos

Passo 4: segurança e padronização do acondicionamento de equipamentos e materiais.

Passo 5: atenção para as especificidades do atendimento pré-hospitalar móvel.

Passo 6: incentivar e valorizar a participação do paciente e família no processo de cuidar. 
Passo 7: promover a comunicação com a central de regulação por meio de radiocomunicação correta ou outras tecnologias, tais como a telemedicina e tele-enfermagem.

Passo 8: prevenção de traumas e quedas; fixar os equipamentos devidamente na ambulância; checar cintos de segurança e travas das macas.

Passo 9: proteger a pele de lesões adicionais provenientes de fricção e cisalhamento; retirar roupa suja e úmida de área de contato com a prancha; evitar contato direto da pele com a prancha rígida.

Passo 10: compreender o benefício e o impacto do uso de todos os equipamentos da ambulância; zelar e verificar o funcionamento das máquinas e equipamentos.

\section{DISCUSSÃO}

A partir da apresentação dos resultados nota-se que, embora dois passos não se apliquem ao APHM (Passo 4 - cirurgia segura e passo 5 - sangue e hemocomponentes - administração segura), outros que se aplicariam não emergiram. Observa-se que os enfermeiros entrevistados não mencionaram diretamente categorias a passos relevantes, tais como: identificação do paciente (Passo 1); paciente envolvido com sua segurança (Passo 6); prevenção de úlcera por pressão (Passo 9); e passo 10: segurança na utilização de tecnologia. Contudo, entende-se que estes passos merecem relevância em qualquer serviço de saúde e verifica-se, portanto, a necessidade de implementar soluções similares que promovam a segurança do paciente no APHM.

Corroborando com a proposta do COREN$-\mathrm{SP},{ }^{10}$ deste estudo emergiram as categorias que igualmente sugestionam passos para a segurança do paciente no APHM: risco de infecção (C3), riscos de traumas e quedas (C4) e riscos para administração de medicamentos (C5), que são referentes, respectivamente, a cuidado limpo e cuidado seguro (Passo 2); prevenção de queda (Passo 8); cateteres e sonda - conexões corretas (Passo 3).

Com relação ao risco de infecção (C3), das 19 combinações de respostas contidas na figura 1 , esta categoria emerge em nove, que corresponde a $47,37 \%$ dos resultados. Nessa premissa, as exposições de riscos de contaminação e infecção, alguns autores mencionam que as superfícies carreiam um risco mínimo de transmissão direta de infecção. Contudo, acontecem secundariamente por meio das mãos dos profissionais de saúde e de instrumentos ou produtos que poderão ser contaminados ao entrar em contato com essas superfícies e, posteriormente, contaminar os pacientes ou outras superfícies. ${ }^{20}$

Remetendo-se a risco de trauma (C4), citado isoladamente por 3,1\% da amostra, e em combinações com outras categorias (31,57\%), evidencia-se na literatura a importância de dimensionar quedas ocorridas com pacientes com vistas à prevenção dos pontos vulneráveis no processo de cuidar implica em considerá-las como eventos passíveis de controle, sendo a atenção fornecida pela equipe às técnicas corretas de segurança, atenuante de possíveis falhas que possam comprometer a segurança do paciente. ${ }^{21}$

Uma das categorias emergidas nos resultados desta pesquisa foi a que refere aos riscos para administração de medicamentos. Sabe-se que a preocupação com o impacto dos erros de medicação na segurança do paciente é da responsabilidade dos profissionais de enfermagem, visando assegurar aos pacientes uma assistência livre de imprudência, imperícia ou negligência. Entretanto, os erros relacionados à administração de medicamentos ocorrem em muitos hospitais e podem trazer sérias consequências para os pacientes, instituição e profissionais. $^{22}$

Podemos definir os erros de medicação como "qualquer incidente previsível que possa causar dano ao paciente ou que dê lugar a uma utilização inapropriada dos medicamentos, quando estes estão sob o controle de profissionais. No geral, esses incidentes estão relacionados com prática a profissional, com os procedimentos ou com os sistemas, incluindo falhas na prescrição, comunicação, etiquetação, envasamento, denominação, preparação, dispensação, distribuição, administração, educação, seguimento e utilização", ${ }^{23}$ de forma que o atendimento pré-hospitalar, com suas peculiaridades quanto à precariedade dos locais de atendimento, à gestão durante ações inesperadas e ao estresse que os profissionais são submetidos, faz com que todas as características do erro descritas acima fiquem mais propensas a acontecer.

Os enfermeiros entrevistados evidenciaram, ainda, a dificuldade no acondicionamento de materiais e equipamentos. Nesse sentido, entende-se que é atribuição do enfermeiro conferir e repor os materiais da ambulância, bem como fazer um checklist diário de equipamentos e de materiais. ${ }^{24}$ Além disso, a padronização dos kits de atendimento e da bolsa de medicamentos, com o propósito de 
facilitar a utilização desses materiais no momento da assistência, também é atribuição do enfermeiro. ${ }^{25}$ Porém, sabe-se que a ambulância é um espaço restrito, fato que dificulta esta organização.

Conforme observa-se nos resultados, a categoria Especificidades do APHM (C2) é citada pelos enfermeiros como fator predisponente a erros. Tais resultados são esperados e justificam-se pelo atendimento pré-hospitalar estar sujeito a fatores ambientais, como mudanças climáticas, riscos de acidente de trânsito e a necessidade de decisões imediatas, fazendo com que o profissional tenha que desenvolver-se constantemente para contornar as adversidades encontradas na sua rotina. Além disso, acrescenta-se a restrição de espaço físico e de tempo. ${ }^{26}$

Ainda podemos pontuar que os profissionais do atendimento pré-hospitalar, devido às condições rudes do ambiente de trabalho, enfrentam situações que os deixam mais vulneráveis a riscos ocupacionais, tais como: acesso difícil às vítimas, insegurança na cena do acidente, realização de procedimentos com o veículo estático ou em movimento, de modo que esses profissionais realizam suas atividades em locais diversificados e, na maioria dos casos, sob condições desfavoráveis de luminosidade, chuva, calor, frio, fluxo de veículos, falta de higiene, presença de animais, pessoas agressivas, tumultos sociais, e tais fatores são condições que diferenciam esse trabalho do realizado no ambiente hospitalar. ${ }^{27}$

Nos eventos adversos de procedimentos invasivos, como no tratamento das vias aéreas no pré-hospitalar, os erros são comuns e potencialmente prejudiciais. Estudos e opiniões destacam outros riscos para a segurança do paciente, como manuseio incorreto de equipamentos, mau funcionamento destes, má gestão médica e desvios de protocolo. Estes fatos frequentemente provocam estresse e sentimento de esgotamento, implicando diretamente na segurança da assistência. ${ }^{25}$

Apesar de neste estudo não terem sido observados riscos relacionados a alguns passos para a segurança do paciente, ${ }^{10}$ sabe-se que atualmente diversas pesquisas retratam um aumento no interesse em envolver o paciente e família no processo de cuidar, a propósito de melhorar a qualidade do cuidado, com vista a aumentar a sua confiança no sistema de saúde. Além disso, o paciente desenvolve um papel ativo e participante na segurança do seu próprio processo de cuidar, ${ }^{28}$ fator importante para um bom prognóstico.

Do mesmo modo, com relação ao Passo 7,10 entende-se que a comunicação clara e efetiva entre os profissionais de saúde deva ser entendida como fator determinante para que não ocorram eventos adversos. Estudos revelam que a comunicação ineficaz, potencializa estes eventos que as crenças e valores do paciente devem ser respeitados e valorizados pelos enfermeiros para a segurança deste. ${ }^{26}$

Outro ponto considerado importante, mesmo que não tenha sido mencionado como risco no APHM, é a prevenção de úlcera por pressão. Nessa premissa, ratifica-se que alguns fatores inerentes ao paciente, tais como condições de imobilização (duração), higiene, com a exposição à sujidade ambiental, além da umidade, contribuem para formação de úlceras de pressão. ${ }^{29}$

Destarte, as melhorias na qualidade dos cuidados de saúde estão ligadas à implementação de uma cultura de segurança nas instituições de saúde e podem ter uma relação direta com a diminuição de eventos adversos e de mortalidade. ${ }^{28}$

\section{CONCLUSÃO}

Observa-se que as adversidades de trabalhar em domicílio, na rua, em detrimento de um ambiente teoricamente controlado como o de um hospital, tornam o meio mais arriscado, seja por fatores ambientais, de trânsito, emocionais, físicos ou de segurança e, portanto, mais propensos a erros. Sendo assim, considera-se que conhecendo os riscos, podemos prevenir comportamentos de erros, fato que gera a segurança necessária no atendimento extra-hospitalar.

Através do reconhecimento destes erros no APHM sugere-se passos para a segurança, baseados nos já pré-existentes nacionalmente como os do COREN-SP, mas que em alguns tópicos não se aplicavam diretamente às rotinas do APHM, como cirurgia segura, administração de hemocomponentes e prevenção de úlcera de pressão.

Com base nas categorias que mais emergiram neste estudo, sugerimos como passos para segurança no APHM: 1) identificar o paciente por meio de pulseira, com cores que sinalizem sua gravidade, principalmente nos casos de múltiplas vítimas. 2) segurança relacionada à prevenção de infecção. 3) segurança na administração de medicamentos. 4) segurança e padronização do acondicionamento de equipamentos e materiais. 5) atenção para as especificidades do atendimento pré-hospitalar móvel. 6) incentivar e valorizar a participação do paciente e família no processo de cuidar. 7) promover a comunicação com a central de regulação, por meio da melhor tecnologia. 8) prevenção de traumas e quedas. 9) proteger a pele de lesões adicionais. 10) 
compreender o benefício e o impacto do uso de todos os equipamentos da ambulância.

Para tanto, faz-se necessário também a implantação de capacitações e educação permanente que estimulem as práticas seguras, bem como a obtenção de recursos tecnológicos facilitadores, como a teleenfermagem e a telemedicina.

Esse estudo vem como um meio de sensibilizar a comunidade científica e instigar para realização de novos estudos voltados para essa temática, visto que o estudo possui limitações por ter sido realizado com um grupo restrito de profissionais, de apenas uma categoria e em um ambiente com contextos e diversidades específicas. Portanto, seria interessante, pela caraterística do atendimento pré-hospitalar, dinâmico e multiprofissional, que o estudo se desse com múltiplas categorias profissionais e em características multicêntricas a fim de estabelecer ações que satisfaçam os níveis de qualidade na segurança do paciente para o atendimento pré-hospitalar como um todo, não esquecendo que por mais que protocolos e ações abrangentes sejam criados, é importante considerar as individualidades e necessidades de cada serviço.

\section{REFERÊNCIAS}

1. Ministério da Saúde (BR). MS e Anvisa anunciam ações para segurança do paciente.2013. Avaliable from: http:// portalsaude.saude.gov.br/portalsaude/ arquivos/pdf/2013/Abr/01/PPT_COLETIVA_ SEGURANCA_PACIENTE_FINAL.pdf. Acesso em: 21 mar. 2017.

2. Celedônio, RM; et al. Políticas de Educação Permanente e Formação em Saúde: uma análise documental. Revista da Rede de Enfermagem do Nordeste, Fortaleza, v. 5, n. 13, p.1100-1110, jan. 2012.

3. Ciconet RM. Atuação e articulação das ações das equipes de suporte básico de um serviço de atendimento móvel de urgência com a central de regulação e as portas da urgência [Internet]. Porto Alegre (RS): Escola de Enfermagem, Universidade Federal do Rio Grande do Sul; 2009 [cited 15 Mar 2012]. Available from: http://www.lume.ufrgs. br/bitstream/handle/10183/19036/000734785. pdf? sequence $=1$

4. Ministério da Saúde (BR). Política Nacional de Atenção às Urgências /Ministério da Saúde. 2. ed. Brasília: Ministério da Saúde; 2004

5. Feldman LB. Como alcançar a qualidade nas instituições de saúde: critérios de avaliações, procedimentos de controle, gerenciamento de riscos hospitalares até a certificação. São Paulo: Martinari; 2004.

6. World Health Organization (WHO). World Alliance for Patient Safety. Research for patient safety: better knowledge for safer care [Internet]. Geneva (Sw); 2008. [cited $2012 \mathrm{Jul}$ 06]. Available from: www.who. int/patientsafety/information_centre/documents/ ps_research_brochure_en.pdf

7. Blegen MA. Patient safety in hospital acute care units. Annu Rev Nurs Res. 2006; 24: 103-25.

8. Gomes AQF. Iniciativas Globais para Segurança do Paciente. Fundação Oswaldo Cruz: Rio de Janeiro: Proqualis; 2010.

9. Pedreira MLG, Harada MJCS, organizadores. Enfermagem dia a dia - segurança do paciente. São Caetano do Sul (SP): Yendis Editora; 2009.

10. Conselho Regional de Enfermagem do Estado de São Paulo (COREN-SP). 10 passos para a segurança do paciente. São Paulo (SP): COREN-SP; 2010. 32 p.

11. Department of Health. About Patient Safety. 2010 [cited 2011 Jun 03]. Available from: http:// www.dh.gov.uk/en/Publichealth/Patientsafety/ Patientsafetygeneralinformation/D H_4066328

12. World Health Organization (WHO). 10 facts on patient safety; 2002. [cited 2011 Nov 19]. Available from: http://www.who.int/features/factfiles/ patient_safety/en/index.html

13. Hagiwara et al. Scandinavian Journal of Trauma, Resuscitation and Emergency Medicine [Internet]. 2016 [cited 2017 Mar 21]; 24(14):1-7. Available from: https://www.ncbi.nlm.nih.gov/pmc/articles / PMC4751749/

14. Bosch J, Nooji J, Visser M, Cannegieter SC, Terpstra NJ, Heringhaus C, et al. Prehospital use in emergency patients of a laryngeal mask airway by ambulance paramedics is a safe and effective alternative for endotracheal intubation. Emerg Med J 2014;31:750-53. Available from: https://www.ncbi.nlm.nih.gov/ pubmed/23771898

15. Hansen M, Meckler G, O'Brien K, Engle P, Dickinson C, Dickinson K, et al. Pediatric Airway Management and Prehospital Patient Safety: Results of a National Delphi Survey by the Children's Safety Initiative-Emergency Medical Services for Children. Pediatr Emerg Care [Internet]. 2016 Sep;32(9):603-7. Available from: https://www.ncbi.nlm.nih.gov/pubmed/27253653

16. Conselho Federal de Enfermagem (Cofen). Resolução COFEN no 389 de 18 de outubro de 2011. Atualiza, no âmbito do Sistema Cofen /Conselhos Regionais de Enfermagem, os procedimentos para registro de título de pós-graduação lato e stricto sensu concedido a enfermeiros e lista as especialidades.

17. Marck P, Cassiani SHB. Teorizando sobre sistemas: uma tarefa ecológica para as pesquisas na área de segurança do paciente. Rev. Latino-Am Enfermagem [Internet]. 2005 [cited $2011 \mathrm{Feb}$ 10]; 13(5):750-3. Available from: http://www.scielo.br/pdf/rlae/ v13n5/v13n5a21.pdf

18. Bardin L. Análise de conteúdo. $4^{\mathrm{a}}$ ed. Lisboa (PT): Edições 70; 2011. 
19. Brasil. Conselho Nacional de Saúde. Resolução $n^{0}$. 466, de 12 de dezembro de 2012. Avaliable from: <http://conselho.saude.gov.br/resolucoes/2012/ Reso466.pdf>. Cited: 21 março 2017.

20. Reis AM, Marques TC, Opitz SP, Silva AE, Gimenes FR, Teixeira TC, et al. Errors in medicine administration profile of medicines: knowing and preventing. Acta Paul Enferm [Internet]. 2010 [cited 2012 Jun 30]; 23 (2):181-6. Available from: http:/ / www.scielo.br/pdf/ ape/v23n2/05.pdf

21. Oliveira RM, Leitão IMTA, Silva LMS, Figueiredo SV, Sampaio R, Gondim MM. Strategies for promoting patient safety: from the identification of the risks to the evidence-based practices. Esc Anna Nery [Internet]. 2014 Mar [cited 2016 May 03]; 18(1):122-9. Available from: http:/ / www.scielo.br/scielo.php?script=sci_ arttext\&pid=S141481452014000100122\&lng=en

22. Richard N. Keers, Steven D. Williams, Jonathan Cooke, Darren M. Ashcroft causes of medication administration errors in hospitals: a systematic review of quantitative and qualitative evidence. Drug Saf [Internet]. 2013 [cited 2016 Mai 03]; 36(11):1045-67. Available from: doi:10.1007/s40264-013-0090-2

23. Santos DS, Souza OV, Nascimento ALS, Pereira JS, Santos MJC, Alves MC, et al. Segurança do Paciente: fatores causais de eventos adversos a medicamentos pela equipe de enfermagem. Ciências Biológicas e da Saúde. 2014; 2( 2): 19-30

24. Mello JF, Barbosa SFF. Patient safety culture in intensive care: nursing contributions. Texto Contexto Enferm. [Internet]. 2013 Dec [cited 2016 May 03]; 22(4):1124-33. Available from: http://www.
scielo.br/scielo.php?script=sci_arttext\&pid=S010407072013000400031\&lng=en

25. Thomaz RR, Lima FV. A atuação do enfermeiro no atendimento pré hospitalar na cidade de São Paulo. Acta Paul Enf [internet]. 2000 [cited 2012 Dec 15]; 13(3):59-65. Available from: http://www.unifesp.br/acta/sum. php? volume $=13 \&$ numero $=3 \&$ item $=$ res7.htm

26. Romazini EM, Bock LF. Conceptions and feelings of nurses working in emergency medical services about their professional practice and training. Rev Latino-Am Enfermagem [Internet]. 2010 [cited 2012 Dec 15]; 18(2):105-12. Available from: http:/ / www. scielo.br/scielo.php?script=sci_arttext\&pid=S010411692010000200015\&lng=en\&nrm=iso\&tlng=en

27. Leite HDCS; Carvalho MTR; Cariman SRL; Araujo ERM; Silva NC; Carvalho AO; Risco ocupacional entre profissionais de saúde do serviço de atendimento móvel de urgência - SAMU. Enferm. Foco. 2016; 7 (3/4): 31-35.

28. Patterson PD, Huang DT, Fairbanks RJ, Simeone $S$, Weaver M, Wang HE. Variation in emergency medical services workplace safety culture. Prehosp Emerg Care [internet]. 2010 [cited 2012 Dec 15]; 14(4):44860. Available from: http://www.ncbi.nlm.nih.gov/ pubmed/20809688

29. Menegon DB, Bercini RR, Santos CT, Lucena AF, Pereira AGS, Scain SF. Braden subscales analysis as indicative of risk for pressure ulcer. Texto Contexto Enferm [Internet]. 2012 [cited 2016 May 03]; 21(4):854-61. Available from: http://www. scielo.br/scielo.php?script=sci_arttext\&pid=S010407072012000400016\&lng=en
Correspondência: Francis Solange Vieira Tourinho Universidade Federal de Santa Catarina - Enfermagem CCS bloco I Sala 501

88015700 - Campus Universitário, Florianópolis, SC, Brasil E-mail: francis.tourinho@ufsc.br
Recebido: 05 de novembro de 2016

Aprovado: 04 de maio de 2017

This is an Open Access article distributed under the terms of the Creative Commons (CC BY). 